\title{
Visual Evoked Potentials Change as Heart Rate and Carotid Pressure Change
}

Barbara B. WALKer

Ann Arbor VA Medical Center and University of Michigan, Ann Arbor

AND CuRT A. SANDMAN

Fairview State Hospital and University of California at Irvine, Costa Mesa

\begin{abstract}
The relationship between cardiovascular activity and the brain was explored by recording visual evoked potentials from the occipital regions of the scalp during systolic and diastolic pressure (Experiment I) and during fast and slow heartbeats at systolic and diastolic pressure (Experiment II). Visual evoked potentials changed significantly as heart rate and carotid pressure fluctuated normally, and these changes were markedly different in the right and left cerebral hemispheres. Evoked potentials recorded from the right hemisphere during various cardiac events differed significantly, whereas those recorded from the left did not. In both experiments, differences in the right hemisphere were due primarily to the P1 component, which was larger at diastolic than at systolic pressure. The present findings are consistent with formulations from behavioral studies suggesting that baroreceptor activity can influence sensory intake, and suggest that hemispheric specialization may play an important role in the relationship between cardiac events, the brain and behavior.
\end{abstract}

DESCRIPTORS: Carotid pressure, Evoked potentials, Heart rate, Baroreceptors.

The early conception that sensory impulses are simply relayed from receptor to cortex was shaken when Granit and Kaada (1952) discovered that the central nervous system could control afferent fibers from muscle spindles. Since then, numerous studies have established that the brain can modulate its own sensory input by inhibiting or facilitating sensory transmission anywhere from the peripheral sense organs to the cortical level. In fact, it has been estimated that $10 \%$ of all afferent fibers contribute to centrifugal control mechanisms (Livingston, 1976), blurring the classical distinction between "sensory" and "motor" mechanisms.

The brainstem reticular formation plays an important role in the centrifugal control of sensory processes. Both electrical stimulation and lesions (Livingston, 1959; Hernandez-Peon, 1961) of the reticular formation can facilitate or block interac-

These studies represent a portion of a doctoral dissertation submitted by the first author in partial fulfillment of the $\mathrm{Ph}$.D. requirements at Ohio State University. Portions of this report were presented to the Society for Psychophysiological Research in Cincinnati, Ohio, 1979. We are grateful to numerous members of the society for their helpful comments during that meeting.

Address requests for reprints to: Barbara B. Walker, Ann Arbor Veterans Administration Medical Center, 116B, 2215 Fuller Road, Ann Arbor, Michigan 48105. tions occurring at numerous levels of sensory transmission. Since the reticular formation is replete with afferent fibers from the viscera, it seems possible that afferent autonomic activity can also serve to modulate sensory input. There is ample reason to speculate that this is the case, since afferent autonomic fibers are known to exert a variety of extra-homeostatic effects. In the case of the cardiovascular system, for example, stimulation of baroreceptors in the carotid sinus leads to decreased motor activity and prolonged sleep (Koch, 1932), shifts in electrocortical activity from low-voltagefast to high-voltage-slow waves (Bonvallet, Dell, \& Hiebel, 1954; Nakao, Ballim, \& Gellhorn, 1956), inhibition of evoked monosynaptic reflexes (Bonvallet, Dell, \& Hugelin, 1954), suppression of sham rage (Bartorelli, Bizzi, Libretti, \& Zanchetti, 1960; Baccelli, Guazzi, Libretti, \& Zanchetti, 1965), depression of pyramidal tract cells in the motor cortex (Coleridge, Coleridge, \& Rosenthal, 1976) and single cell firing to skin stimulation in the nucleus cuneatus (Gahery \& Vigier, 1974), and diminished sweat gland and nictitating membrane activity in cats (Horowitz \& Kaufman, 1979).

It has also been proposed that the inhibition resulting from increases in baroreceptor firing is behaviorally significant (Lacey, 1967). Cardiac acceleration is associated with slower reaction times and 
impaired visual perception, whereas cardiac deceleration is associated with faster reaction times and enhanced visual perception (Lacey, Kagan, Lacey, \& Moss, 1963; Lacey, 1967; Lacey, 1972; DuncanJohnson \& Coles, 1974; Sandman, McCanne, Kaiser, \& Diamond, 1977). Attempts to relate behavioral changes to differential firing of baroreceptors within a cardiac cycle, however, have yielded equivocal results. Even when faster reaction time or enhanced visual perception has been found early in the cardiac cycle (when baroreceptors fire minimally) compared to late (when baroreceptors fire maximally), the effects have not been robust (Birren, Carden, \& Phillips, 1963; Callaway \& Layne, 1964; Sandman et al., 1977).

In view of these data and the controversy surrounding the behavioral significance of cardiac events (Elliott, 1972; Lacey \& Lacey, 1974b; Carroll \& Anastasiades, 1978; Obrist, 1976), it is surprising that so few studies have examined the relationship between cardiac events and central nervous system activity in human subjects. Early studies suggested that cardiac events were related to electroencephalographic (EEG) activity (Callaway, 1965; Callaway \& Buchsbaum, 1965; Callaway \& Layne, 1964), but details of the relationship are difficult to ascertain from these reports. More recently, an experiment from our laboratory demonstrated that average evoked potentials (AEPs) changed as heart rate fluctuated normally (Walker \& Sandman, 1979). In this experiment, changes in heart rate were related to the right and left cerebral hemispheres differently, suggesting that hemispheric specialization may play some role in the relationship between cardiac events and behavior.

The following experiments were designed to further explore the relationship between cardiac activity and the brain. If baroreceptors are involved in the relationship between cardiac events and behavior, then AEPs recorded at systolic (when baroreceptors fire maximally) and diastolic pressure (when baroreceptors fire minimally) should differ. Specifically, one would expect AEPs to be smaller during systolic pressure than during diastolic pressure due to the inhibitory effects of baroreceptor stimulation.

\section{EXPERIMENT I}

\section{Visual Evoked Potentials and Carotid Pressure ${ }^{1}$}

\section{Method}

\section{Subjects}

Subjects who volunteered to participate in the study as part of a requirement for Introductory Psychology classes at Ohio State University were screened and 9 men

'Some of the data presented here are also discussed in a recent book chapter (Sandman, Walker, \& Berka, 1982). and 9 women who met the following criteria were selected to participate: $20 / 20$ corrected vision, righthanded, between the ages of 18 and 25 , absence of drug usage, and no cardiovascular pathology or sleep difficulties.

\section{Procedure}

Subjects reclined in a comfortable chair while transducers were attached for recording heart rate, respiration, EEG, eye movements, and carotid pulse pressure. Care was taken to ensure that each subject was in a position that did not entail continuous postural tension. Subjects wore headphones with masking noise to eliminate all extraneous auditory stimuli. A dot was positioned on the screen in front of each subject and subjects were instructed to relax, breathe regularly, and to keep their eyes fixated on the dot at all times. Three rest periods were given during each session; these were signified by turning the masking noise off for a few minutes.

\section{Apparatus}

The subjects were tested in a sound-attenuating and electrically shielded room. Recording, programming, and stimulus presentation equipment were housed in an adjacent room. Physiological recordings were obtained with a Grass Model 7B polygraph equipped with the appropriate preamplifiers and driver amplifiers. In addition to being recorded on the polygraph, both channels of EEG (right and left hemispheres) were recorded on a four-channel Sony FM tape recorder to enable off-line computer analyses of these data. The carotid pressure wave was transmitted to two comparators set to detect the highest and lowest points of the wave (systolic and diastolic pressure). When criterion was met, a $20-\mathrm{msec}$ flash was projected on the screen by a Kodak projector tachistoscope. A minimum of $3 \mathrm{sec}$ elapsed between flashes. Each stimulus was marked on both the tape and the polygraph. Each subject received 50 flashes during systolic and 50 during diastolic pressure in a semi-random order.

Carotid Pressure. A photoplethysmograph was taped over the carotid artery. This photoplethysmograph contained a light-emitting diode (LED) and a narrow band emitter of infrared radiation with a wavelength of .74 micrometers. The emitter of the plethysmograph was in the same plane as a photodarlington resistor of matched sensitivity, and the two devices were mounted on a small glass epoxy printed circuit. The signal was amplified using a time constant of .08 and a half-amplitude high frequency of $15 \mathrm{~Hz}$. This provided a very clear recording of carotid pressure with few artifacts other than those associated with swallowing. Stimuli presented at these times were deleted from all analyses.

$E E G$. Sites designated by the International $10-20$ system (Jasper, 1958) as $\mathrm{O}_{1}$ and $\mathrm{O}_{2}$ and both mastoids were rubbed briskly with acetone to reduce resistance. Ag$\mathrm{AgCl}$ electrodes filled with Grass EEG Creme were then affixed to the scalp with collodion and linked from $\mathrm{O}_{1}$ to the left mastoid and $\mathrm{O}_{2}$ to the right mastoid to permit unipolar recordings from the right and left occipital cortex. Pairs of electrodes showing more than $10 \mathrm{~K}$ ohms impedance were replaced. The signal was amplified using a 
half-amplitude low frequency setting of $1 \mathrm{~Hz}$ (corresponding to a time constant of $.1 \mathrm{sec}$ ) and a half-amplitude high frequency setting of $35 \mathrm{~Hz}$ (Goff, 1974).

Heart Rate. Grass Ag-AgCl cup electrodes filled with EKG Sol were attached to the area of the lower left rib and the right collar bone of each subject after these areas were swabbed with acetone. The signal was amplified by a Grass AC preamplifier and processed by a cardiotachometer to provide beat-by-beat heart rate recordings.

Respiration. The respirometer consisted of a thinwalled opaque neoprene tubing $5.1 \mathrm{~cm}$ in length and 3.5 $\mathrm{mm}$ in diameter with an optical sensor and emitter mounted at each end. The mounting was roughly spherical with a diameter such that the tubing, when stretched across the chest, was drawn taut in a straight line between mounts. Attached to each mount was a cloth band 1.95 $\mathrm{cm}$ in width and of sufficient length to encompass different chest sizes. Velcro fasteners on each end held the respirometer in place. Current output of the phototransistor was approximately linear with chest expansion. The varying voltage was amplified by a Grass AC preamplifier.

Eye Movements. Ag- $\mathrm{AgCl}$ electrodes were placed next to and below the left eye about $2 \mathrm{~cm}$ from the orbital cavity using Grass EKG Sol. The purpose of this measure was to enable detection of all trials involving eye movements or eye blinks.

\section{Data Reduction}

Average Evoked Potentials. Electrocortical activity associated with the 50 flashes presented at systolic and diastolic pressure was converted from an analog to a digital signal at a sampling rate of $100 \mathrm{~Hz}$ for a period of 500 msec following each flash. The AEPs associated with systolic and diastolic pressure were then averaged across subjects and graphed using a Nova $3 / 12$ computer system. This yielded two AEPs recorded from each hemisphere for each subject. Latencies and amplitudes ${ }^{2}$ of the positive peaks occurring approximately 40 (primary component), 100 (P1), 200 (P2), and 300 (P3) msec following the flash were calculated for the four AEPs recorded from each subject. Since identification of prominent peaks in the AEP is sometimes difficult due to variability, two independent judges chose the peaks in each AEP. The mean of the interrater reliability coefficients computed for each subject was .96 , indicating that the four peaks were represented clearly in all but a few of the waveforms obtained.

Respiration. Frequency and depth of respiration were monitored but not subjected to analysis. Flashes associated with changes in respiration were repeated. Few such occurrences were noted.

Eye Movements. Trials associated with eye movements or eye blinks were repeated and deleted from all analyses.

\section{Results}

The AEPs recorded from the right and left occipital regions during systolic and diastolic pressure

${ }^{2}$ Amplitudes were calculated by finding the difference (in microvolts) between the positive peak and the negative peak that preceded it.

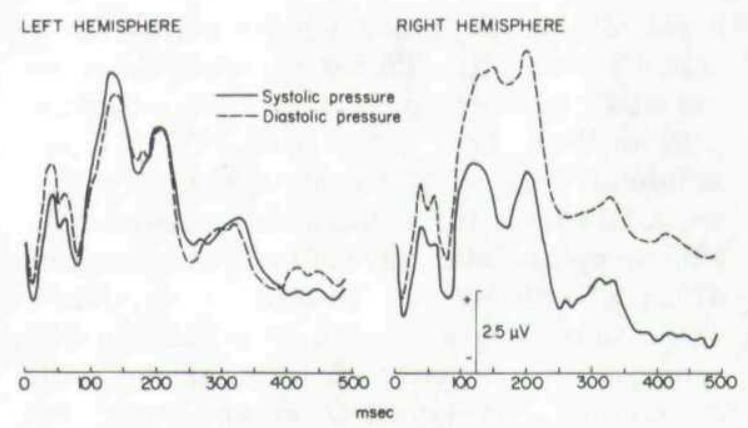

Figure 1. Visual evoked potentials recorded from the right and left hemispheres at systolic and diastolic pressure.

are shown in Figure 1. As shown in the figure, AEPs changed as carotid pressure changed, and these changes differed in the two hemispheres. A 2 (systolic/diastolic) $\times 2$ (hemispheres) $\times 4$ (AEP components) analysis of variance indicated a significant three-way interaction for amplitudes, $F(3 /$ $51)=8.70, p<.01$. With the data collapsed across carotid pressure, AEPs recorded from the right and left hemispheres did not differ. The two hemispheres, however, were related to carotid pressure differently. In the right hemisphere, P1 was larger at diastolic pressure, but in the left hemisphere, P1 was larger at systolic pressure. This difference was statistically significant in the right hemisphere, $F(1 /$ 17) $=4.90, p<.05$, but not in the left. There were no consistent differences among latencies.

It was of interest to determine the extent to which these results were represented in each individual subject. Therefore, the P1 amplitudes in the AEPs recorded from each subject were examined. In the right hemisphere, P1 amplitudes were larger at diastolic than at systolic pressure in 14 of the 18 subjects, which was statistically significant (Sign Test: $p<.01$; Wilcoxon $T$ test: $T=27, p<.01$ ). In the left hemisphere, P1 was larger at systolic pressure in the majority (12) of subjects, but this was not statistically significant.

\section{Discussion}

Changes in carotid pressure were clearly related to changes in visual evoked potentials. Similar to previous findings with heart rate (Walker \& Sandman, 1979), these changes were markedly different in the two hemispheres. In the right hemisphere, AEPs recorded during systolic and diastolic pressure differed significantly, whereas in the left hemisphere this was not the case. The differences in the right hemisphere were due primarily to the P1 component, which was larger during diastolic pressure. Since both the P1 component (Beck, 1975; Dustman, Schenkenberg, \& Beck, 1976; Van Voorhis \& Hillyard, 1977) and the right hemisphere have been related to attention (Dustman et al., 1976; Heilman \& Abell, 1980), these differences are con- 
sistent with the idea that increases and decreases in baroreceptor activity can impair or facilitate sensory intake (Lacey, 1967; Lacey \& Lacey, 1974a). Furthermore, the fact that cardiac events were related to the right and left hemispheres differently suggests that hemispheric specialization may be an important variable that has been ignored in behavioral studies of cardiac-behavioral relationships to date.

These findings and those reported earlier regarding heart rate indicate that visual evoked potentials change as heart rate and carotid pressure fluctuate normally. In an intact, healthy organism, however, heart rate and carotid pressure do not influence baroreceptors independently. Baroreceptors respond to distortion, and therefore they encode interactions between carotid pressure and heart rate. The way that baroreceptors encode these interactions is complex and the details are as yet unclear (Ead, Green, \& Neil, 1952; Gero \& Gerova, 1967; Arndt, Morgenstern, \& Samodelev, 1977; Brown, 1980), but investigators agree that the impact that systolic and diastolic pressure exert on the carotid sinus depends, in part, upon heart rate (see Brown, 1980).

The significance of this interaction has been demonstrated in behavioral studies as well as in studies of baroreceptors. For example, the relationship between heart rate and reaction time is greater when stimuli are presented during diastole than during systole, and as heart rate increases, subjects press a key later and later within a cardiac cycle (Lacey \& Lacey 1974a, 1978). While interpretation of these data has been somewhat difficult (see Lacey \& Lacey, 1978), it is clear that the behavioral changes that occur within a cardiac cycle, like changes in baroreceptor activity, are somewhat dependent upon heart rate.

The following experiment was designed to examine this interaction with respect to electrocortical activity. Although its significance has been demonstrated for both baroreceptors and behavior, the electrocortical changes that occur as heart rate and carotid pressure covary have not yet been explored. Examination of such changes may shed light on the complex set of findings that have emerged regarding the impact of this interaction upon both baroreceptors and behavior.

\section{EXPERIMENT II \\ Visual Evoked Potentials, Heart Rate, and Carotid Pressure}

\section{Method \\ Subjects}

Fifteen right-handed men who had variable heart rates and met the same criteria as those who participated in Experiment I served in the experiment.

\section{Procedure}

The procedure was similar to the one used in the first experiment except that 50 flashes of light were presented during each of the following four conditions: a) fast heartbeats/systolic pressure, b) fast heartbeats/diastolic pressure, c) slow heartbeats/systolic pressure, and d) slow heartbeats/diastolic pressure. Voltage changes associated with carotid pressure and heart rate (obtained from the output of the tachograph) were detected using a BRS digibit logic system and comparators. When criteria were met for the predetermined heart rate $^{3}$ and carotid pressure levels, a 20 -msec flash was projected on the screen. As in the first experiment, a minimum of $3 \mathrm{sec}$ elapsed between flashes, and stimuli associated with the four conditions were presented in a semi-random order. Heart rate, carotid pressure, eye movements, respiration, and EEG were monitored as described earlier.

\section{Data Reduction}

Average Evoked Potentials. The electrocortical activity associated with the 50 flashes presented during each of the four conditions was converted from an analog to a digital signal at a sampling rate of $100 \mathrm{~Hz}$ for a period of $500 \mathrm{msec}$ following the flash. These data were then averaged and graphed (using a Nova $3 / 12$ computer system), yielding four AEPs for each subject for each hemisphere. As described earlier, latencies and amplitudes were computed for each of the eight AEPs obtained for each subject.

Heart Rate. The heart rates of five beats were recorded for each flash: two heartbeats prior to the stimulus, the beat during which the stimulus occurred, and the two beats following the stimulus.

Eye Movements and Respiration. Trials associated with eye movements, eye blinks, or changes in respiration were repeated and deleted from all analyses. No further analyses were conducted.

\section{Results}

\section{Heart Rate}

To test whether the flashes of light actually occurred at different heart rates, a 4 (conditions) $\times 5$ (blocks) $\times 10$ (flashes) $\times 5$ (heartbeats) analysis of variance with repeated measures of all the factors was performed. This analysis revealed a highly significant interaction between conditions and beats, $F(12 / 168)=37.31, p<.01$. The mean heart rate that elicited stimuli for the fast heartbeats/systolic condition $(75.5 \mathrm{bpm})$ was almost identical to the heart rate level for the fast heartbeats/diastolic condition $(75.0 \mathrm{bpm})$, but these differed significantly from heart rate during slow heartbeats/systolic

${ }^{3}$ Criteria for slow and fast heartbeats were defined as $10 \%$ slower and $10 \%$ faster than the heart rate that was calculated after the transducers were attached. Since average heart rates decelerated from the beginning to the end of the session, these criteria were adjusted several times during the experiment to ensure maximal differences between fast and slow heartbeats. 
$(65.3 \mathrm{bpm})$ and slow heartbeats/diastolic conditions $(65.3 \mathrm{bpm})$.

\section{Carotid Pressure, Heart Rate, and the AEP}

To test whether the impact of systolic and diastolic pressure differed during fast and slow heartbeats, the AEPs associated with each of the four conditions were averaged for all subjects for both hemispheres separately. This yielded four AEPs recorded from the right hemisphere and four recorded from the left hemisphere; these are illustrated in Figure 2.

Amplitudes and latencies were first compared using a 2 (heart rates) $\times 2$ (systolic/diastolic) $\times 2$ (hemispheres) $\times 4$ (AEP components) analysis of variance. Similar to findings in the first experiment, with the data collapsed across heart rate and carotid pressure, there were no differences between AEPs recorded from the right and left hemispheres. A significant 3-way interaction between pulse pressure, hemisphere, and components, $F(3 /$ $42)=5.91, p<.01$, emerged, however, indicating differences among amplitudes. P1 was larger in the right hemisphere during diastolic pressure but larger in the left hemisphere during systolic pressure. To further characterize these differences, four 2 (systolic/diastolic) $\times 4$ (AEP components) analyses of variance were performed to compare the amplitudes shown in each quadrant of Figure 2. Only the two AEPs recorded from the right hemisphere during high heart rate differed significantly, $F(3 / 42)=5.33, p<.01$. The amplitude of $\mathrm{P} 1$ was greater at diastolic pressure than at systolic pressure, $F(1 / 14)=18.21, p<.01$. As in the first experiment, latencies did not differ significantly.

As indicated earlier, it was important to determine the extent to which the averaged data represented individual subjects. In AEPs recorded from the right hemisphere during fast heartbeats, P1 was

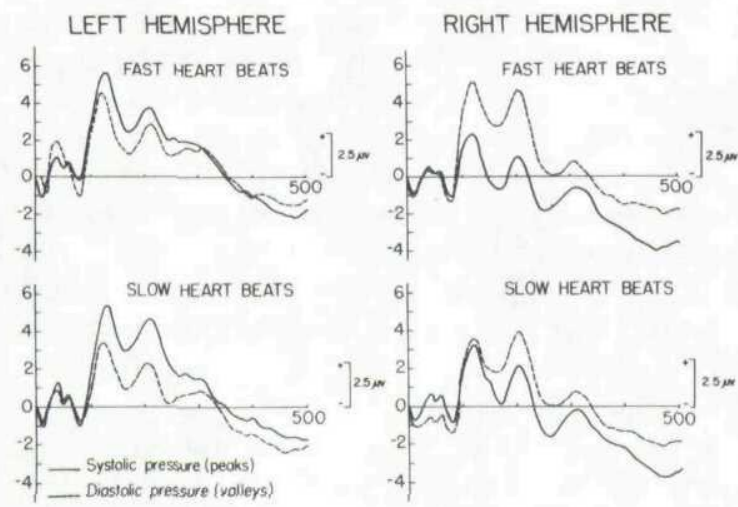

Figure 2. Visual evoked potentials recorded as heart rate and carotid pressure covary. larger at diastolic pressure in 12 of the 15 subjects (Sign Test: $p<.02$; Wilcoxon $T$ test: $T=6, p<.01$ ), whereas during slow heartbeats, P1 was larger during diastolic pressure in only 9 of the subjects, which was nonsignificant. In AEPs recorded from the left hemisphere during fast heartbeats, P1 was larger during systolic pressure in only 7 subjects, whereas during slow heart beats, P1 was larger during systolic pressure in 12 subjects (Sign Test: $p<.02$; Wilcoxon $T$ Test: $T=22, p<.05$ ). It thus appears that diastolic pressure exerts its greatest influence on the right hemisphere during fast heartbeats and that systolic pressure exerts its greatest impact on the left hemisphere during slow heartbeats. The differences in the left hemisphere should be interpreted cautiously, however, since these differences were not statistically significant using the analysis of variance.

\section{Discussion}

As reviewed earlier, baroreceptors do not respond to changes in heart rate and pulsatile pressure independently. The two interact, and it is clear from the present study that this interaction is related to changes in the visual evoked response as well as to changes in baroreceptors, reaction time, and perception. Similar to findings reported in Experiment I, P1 was larger in the right hemisphere at diastolic pressure than at systolic pressure, but the reverse was true in the left hemisphere. Furthermore, differences between AEPs recorded from the right hemisphere during systolic and diastolic pressure were much greater during fast heartbeats.

In view of these findings, it is not surprising that attempts to relate cardiac phase to behavior have yielded equivocal results. First, as discussed earlier, the present findings suggest that the relationship between the heart and the brain is lateralized, and this factor has not been considered in behavioral studies. Second, the present study indicates that the effects of cardiac phase depend upon heart rate. Some investigations of cardiac phase and behavior have used reaction time tasks with a warning signal (Thompson \& Botwinick, 1970; Delfini \& Campos, 1972) which leads to cardiac deceleration (Graham \& Clifton, 1966; Lacey \& Lacey, 1970; Lacey, 1972). The present findings suggest that the influence of changes within one heartbeat on behavior may fade with cardiac deceleration, which might explain why some studies using a warning signal have not yielded significant results (Thompson \& Botwinick, 1970; Delfini \& Campos, 1972), whereas others not using a warning signal have found significant relationships (Saari \& Pappas, 1976; Sandman et al., 1977). 


\section{GENERAL DISCUSSION}

The impact that stimuli exert on the brain varies as heart rate and carotid pressure vary. One explanation for these differences is that cells in the brain that control cardiovascular activity simultaneously influence electrocortical activity. This seems plausible with respect to changes in AEPs associated with heart rate, but it is difficult to reconcile with the dramatic changes in AEPs found during systolic and diastolic pressure. In addition, very few cells have been found that fire at a cardiac rhythm (Smith \& Pearce, 1961; Humphrey, 1967), and there is no evidence that these changes are represented at the cortical level. Finally, the occipital region of the brain has never been implicated in cardiovascular control (Uvnas, 1960), and it seems unlikely that higher cortical activity would show hemispheric specialization with regard to a vital function such as cardiac control.

A more parsimonious explanation is that cardiovascular changes that are encoded by baroreceptors and transmitted to the nucleus tractus solitarius in the reticular formation (Bonvallet \& Bloch, 1961; Bonvallet \& Allen, 1963) modulate the impact stimuli exert on the brain. This explanation is consistent with the mounting evidence that afferent fibers from the cardiovascular system influence central neuronal activity that is unrelated to cardiovascular control (see Horowitz \& Kaufman, 1979). It would also account for the fact that AEPs recorded during different heart rates and carotid pressures were similar but not identical, and that they varied in a manner that could be predicted based on studies of baroreceptors.

The fact that cardiac events were related to the right and left hemispheres differently suggests a new dimension to the study of relationships between cardiac activity and the brain..$^{4}$ In the present

${ }^{4}$ It is important to note a few aspects of our recording procedures that could have contributed to this lateralization. First, the plethysmograph was usually placed over the left carotid artery, which may have provided lateralized kinesthetic feedback and influenced the AEPs (Lacey, Note 1). AEPs recorded at different heart rates and pulse pressures recorded from the right supraorbital artery, however, have shown similar lateralization (Walker, 1979), which argues against this as the sole explanation. Second, mastoids were not linked in these studies, and cardiac events may influence the right and left mastoids differently, leading to differences in AEPs recorded from the two hemispheres. This is currently under investigation in our laboratory. Finally, since the heart is asymmetrical, electrical activity from the heart could spread asymmetrically to the two hemispheres and summate with the evoked potentials. In future experiments, studies, AEPs recorded from the right hemisphere were largest at diastolic pressure and those recorded from the left hemisphere were largest at systolic pressure. Both the right hemisphere (Dustman et al., 1976; Heilman \& Abell, 1980) and transient bradycardia (Lacey, 1967) have been related to attention, and the left hemisphere (Donchin, McCarthy, \& Kutas, 1977) and brief hypertensive states (Lacey, 1967) have been associated with cognitive processing. Although behavioral measures were not taken in these experiments, it is tempting to speculate that the relationship between cardiac activity and behavior may involve differential stimulation of the two cerebral hemispheres.

Although the mechanisms are as yet unclear, there are several factors that could contribute to this lateralization. The heart, like the brain, is not a symmetrical organ and there are considerable anatomic and physiologic differences between the right and left carotid arteries (DeGeest, Levy, Zieske, \& Lipman, 1965; Berne \& Levy, 1967). In addition, some baroreceptors are situated in the aortic arch and innervation of the aortic arch is lateralized (see Netter, 1962, 1978). Baroreceptor impulses from both the carotid sinus and aortic arch are transmitted to the nucleus tractus solitarius where they associate with the reticular activating system which can influence the two hemispheres differently (Starzl \& Magoun, 1951; French, 1960). Finally, the changes in AEPs may be related to changes in regional blood flow that have been associated with various types of mental activity and electrocortical changes (Ingvar, 1971, 1977; Risberg, Halsey, Wills, \& Wilson, 1975).

Since the present studies were correlational, the mechanisms remain speculative. What is clear from these studies, however, is that normal fluctuations in cardiac activity coincide with changes in the context with which the two cerebral hemispheres receive information. These changes are consistent with physiological studies of baroreceptors and formulations from behavioral studies suggesting that changes in baroreceptor activity influence sensory intake. In addition, these findings suggest that hemispheric specialization may play some role in the relationships between cardiac events, the brain and behavior, and should be considered in future experiments.

it will be important to record the electrocortical activity that is time-locked to the cardiac event under investigation and perhaps subtract this from the actual AEPs. Preliminary data from our laboratory, however, have not revealed any lateralized electrocortical activity that is time-locked to the carotid pressure wave. 


\section{REFERENCES}

Arndt, J. O., Morgenstern, J., \& Samodelev, L. The physiologically relevant information regarding systemic blood pressure encoded in the carotid sinus baroreceptor discharge pattern. Journal of Physiology, 1977, 268, 775-791.

Baccelli, G., Guazzi, M., Libretti, A., \& Zanchetti, A. Pressoceptive and chemoceptive aortic reflexes in decorticate and in decerebrate cats. American Journal of Physiology, 1965, 208, 708-714.

Bartorelli, C., Bizzi, E., Libretti, A., \& Zanchetti, A. Inhibitory control of sinocarotid pressoceptive afferents on hypothalamic autonomic activity and sham rage behavior. Archives Italiennes de Biologie, 1960, 98, 308-326.

Beck, E. Electrophysiology and behavior. Annual Review of Psychology, 1975, 26, 233-262.

Berne, R., \& Levy, M. Cardiovascular physiology. St. Louis: C. V. Mosby, 1967. Pp. 132-169.

Birren, J., Cardon, P., \& Phillips, S. Reaction time as a function of the cardiac cycle in young adults. Science, 1963, 140, 195-196.

Bonvallet, M., \& Allen, M. Prolonged spontaneous and evoked reticular activation following discrete bulbar lesions. Electroencephalography \& Clinical Neurophysiology, 1963, 15, 969-988.

Bonvallet, M., \& Bloch, V. Bulbar control of cortical arousal. Science, 1961, 133, 1133-1134.

Bonvallet, M., Dell, P., \& Hiebel, G. Tonus sympathetique et activite electrique cortical. Electroencephalography \& Clinical Neurophysiology, 1954, 6, 119-144.

Bonvallet, M., Dell, P., \& Hugelin, A. Influence de l'adrenaline sur le controle reticulaire des activites cortical et spinale. Journal de Physiologie (Paris), 1954, 46, 262-265.

Brown, A. Receptors under pressure: An update on baroreceptors. Circulation Research, 1980, 46, 1-10.

Callaway, E. Response speed, the EEG alpha cycle, and the autonomic cardiovascular cycle. In A. T. Welford \& J. E. Birren (Eds.), Behavior, aging and the nervous system. Springfield: C C Thomas, 1965. Pp. 217-233.

Callaway, E., \& Buchsbaum, M. Effects of cardiac and respiratory cycles on averaged visual evoked potentials. Electroencephalography \& Clinical Neurophysiology, 1965, 19, 476-480.

Callaway, E., \& Layne, R. Interaction between the visual evoked response and two spontaneous biological rhythms: The EEG alpha cycle and the cardiac arousal cycle. Annals of the New York Academy of Sciences, $1964,112,424-431$.

Carroll, D., \& Anastasiades, P. The behavioral significance of heart rate: The Laceys' hypothesis. Biological Psychology, 1978, 7, 249-275.

Coleridge, H. M., Coleridge, J. C., \& Rosenthal, F. Prolonged inactivation of cortical pyramidal tract neurones in cats by distension of the carotid sinus. Journal of Physiology, 1976, 256, 635-649.

DeGeest, H., Levy, M., Zieske, H., \& Lipman, R. Depression of ventricular contractibility by stimulation of the vagus nerves. Circulation Research, 1965, 17, 222-234.

Delfini, L., \& Campos, J. Signal detection and the "cardiac arousal cycle." Psychophysiology, 1972, 9, 484-491.
Donchin, E., McCarthy, G., \& Kutas, M. Electroencephalographic investigations of hemispheric specialization. In J. E. Desmedt (Ed.), Progress in clinical neurophysiology (Vol. 3). New York: S. Barger, 1977. Pp. 212-243.

Duncan-Johnson, C., \& Coles, M. Heart rate and disjunctive reaction time: The effects of discrimination requirements. Journal of Experimental Psychology, 1974, 103, 1160-1168.

Dustman, R. E., Schenkenberg, T., \& Beck, E. C. Development of the evoked response as a diagnostic and evaluative procedure. In R. Karrer (Ed.), Developmental psychophysiology of mental retardation. Springfield: C C Thomas, 1976. Pp. 247-310.

Ead, H. W., Green, J. H., \& Neil, E. Comparison of the effects of pulsatile and non-pulsatile blood flow through the carotid sinus on the reflexogenic activity of the sinus baroreceptors in the cat. Journal of Physiology, 1952, 118,509 .

Elliott, R. The significance of heart rate for behavior: A critique of Lacey's hypothesis. Journal of Personality \& Social Psychology, 1972, 22, 398-409.

French, J. D. The reticular formation. In J. Field (Ed.), Handbook of physiology (Sec. I). Washington: American Physiological Society, 1960. Pp. 1281-1305.

Gahery, Y., \& Viegier, D. Inhibitory effects in the cuneate nucleus produced by vago-aortic afferent fibers. Brain Research, 1974, 75, 241-246.

Gero, J., \& Gerova, M. Significance of the individual parameters of pulsating pressure in stimulation of baroreceptors. In P. Kezdi (Ed.), Baroreceptors and hypertension. New York: Pergamon Press, 1967. Pp. 17-31.

Goff, W. R. Human average evoked potentials: Procedures for stimulating and recording. In R. F. Thompson \& M. M. Patterson (Eds.), Bioelectric recording techniques (Part B). New York: Academic Press, 1974. Pp. 102-156.

Graham, F. K., \& Clifton, R. K. Heart rate change as a component of the orienting response. Psychological Bulletin, 1966, 65, 305-320.

Granit, R., \& Kaada, B. R. Influence of stimulation of central nervous structures on muscle spindles in cat. Acta Physiologica Scandinavica, 1952, 27, 130-160.

Heilman, K. M., \& Abell, T. Right hemisphere dominance for attention: The mechanism underlying hemispheric asymmetries of inattention (neglect). Neurology, 1980, 30, 327-330.

Hernandez-Peon, R. Reticular mechanisms of sensory control. In W. Rosenblith (Ed.), Sensory communication. New York: John Wiley, 1961. Pp. 497-520.

Horowitz, I. S., \& Kaufman, A. I. Effects of baroreceptor activation on spontaneous activity in the sweat glands and nictitating membrane of the cat. Brain Research, 1979, $179,411-424$.

Humphrey, D. R. Neuronal activity in the medulla oblongata of cat evoked by stimulation of the carotid sinus nerve. In P. Kezdi (Ed.), Baroreceptors and hypertension. New York: Pergamon Press, 1967. Pp. 131-168.

Ingvar, D. H. Cerebral blood flow and metabolism related to EEG and cerebral functions. Acta Anaesthesiologica Scandinavica, 1971, 39, 110-114.

Ingvar, D. H. Linkage between cerebral blood flow and neu- 
rological function in man. International Journal of Neurology, 1977, 11, 118-128.

Jasper, H. H. Report of the Committee of Clinical Examination in Electroencephalography. Electroencephalography \& Clinical Neurophysiology, 1958, 10, 370-375.

Koch, E. Die Irradiation der pressoreceptorischen kreislaufreflexe. Klinische Wochenschrift, 1932, 11, 225-227.

Lacey, B. C., \& Lacey, J. I. Two-way communication between the heart and the brain: Significance of time within the cardiac cycle. American Psychologist, 1978, 33, 99-113.

Lacey, B. C., \& Lacey, J. I. Studies of heart rate and other bodily processes in sensorimotor behavior. In P. A. Obrist, A. H. Black, J. Brener, \& L. V. DiCara (Eds.), Cardiovascular psychophysiology-Current issues in response mechanisms, biofeedback and methodology. Chicago: Aldine, 1974. Pp. 538-564. (a)

Lacey, J. I. Somatic response patterning and stress: Some revisions of activation theory. In M. H. Appley \& R. Trumbull (Eds.), Psychological stress: Issues in research. New York: Appleton-Century-Crofts, 1967. Pp. 14-44.

Lacey, J. I. Some cardiovascular correlates of sensorimotor behavior: Examples of visceral afferent feedback. In C. B. Hockman (Ed.), Limbic system mechanisms and autonomic function. Springfield, 11.: C C Thomas, 1972. Pp. 175-196.

Lacey, J. I., Kagan, J., Lacey, B. C., \& Moss, H. A. The visceral level: Situational determinants and behavioral correlates of autonomic response patterns. In P. G. Knapp (Ed.), Expression of the emotions in man. New York: International Universities Press, 1963. Pp. 161-196.

Lacey, J. I., \& Lacey, B. C. Some autonomic-central nervous system interrelationships. In P. Black (Ed.), Physiological correlates of emotion. New York: Academic Press, 1970. Pp. 205-228.

Lacey, J. I., \& Lacey, B. C. On heart rate responses and behavior: A reply to Elliott. Journal of Personality and Social Psychology, 1974, 30, 1-18. (b)

Livingston, R. B. Central control of receptors and sensory transmission systems. In J. Field, H. Morgan, \& V. Hall (Eds.), Handbook of physiology (Sec. I, Vol. I). Neurophysiology. Washington: American Physiological Society, 1959. Pp. 741-760.

Livingston, R. B. Sensory processing, perception and behavior. In R. Grenell \& S. Gabay (Eds.), Biological foundations of psychiatry. New York: Raven Press, 1976. Pp. 47-145.

Nakao, H., Ballim, H., \& Gellhorn, E. The role of the sinoaortic receptors in the action of adrenaline, noradrena- line and acetylcholine on the cerebral cortex. Electroencephalography \& Clinical Neurophysiology, 1956, 8, 413-420.

Netter, F. H. Ciba Collection of Medical Illustrations. The nervous system (Vol. I). New York: Ciba Pharmaceutical Co., 1962. Pp. 84-85.

Netter, F. H. Ciba Collection of Medical Illustrations. Heart. New York: Ciba Pharmaceutical Co, , 1978. P. 75.

Obrist, P. A. The cardiovascular-behavior interaction-As it appears today. Psychophysiology, 1976, 13, 95-107.

Risberg, J., Halsey, J. H., Wills, E., \& Wilson, E. M. Hemispheric specialization in normal man studied by bilateral measurements of the regional cerebral blood flow. Brain, 1975, 98, 511-524.

Saari, M., \& Pappas, B. Cardiac cycle phase and movement and reaction times. Perceptual \& Motor Skills, 1976, 42, $767-770$.

Sandman, C. A., McCanne, T. R., Kaiser, D. N., \& Diamond, B. Heart rate and cardiac phase influences on visual perception. Journal of Comparative \& Physiological Psychology, 1977, 91, 189-202.

Sandman, C. A., Walker, B. B., \& Berka, C. Influence of afferent cardiovascular feedback on behavior and the cortical evoked potential. In J. T. Cacioppo \& R. E. Petty (Eds.), Perspectives in cardiovascular psychophysiology. New York: Guilford Press, 1982. Pp. 189-222.

Smith, R. E., \& Pearce, J. W. Microelectrode recordings from the region of the nucleus solitarius in the cat. Canadian Journal of Biochemical Physiology, 1961, 39, 933-939.

Starzl, T. E., \& Magoun, H. W. Organization of diffuse thalamic projection system. Journal of Neurophysiology, $1951,14,133$.

Thompson, L., \& Botwinick, J. Stimulation in different phases of the cardiac cycle and reaction time. Psychophysiology, 1970, 7, 57-65.

Uvnas, B. Central cardiovascular control. In J. Field, H. Magoun, \& V. Hall (Eds.), Handbook of physiology (Sec. I, Vol. II). Washington: American Physiological Society, 1960. Pp. 1131-1162.

VanVoorhis, S., \& Hillyard, S. Visual evoked potentials and selective attention to points in space. Perception and Psychophysics, 1977, 22, 54-62.

Walker, B. B. The emergence of behavior from integrated patterns of central and autonomic nervous system activity. Unpublished doctoral dissertation, Ohio State University, 1979.

Walker, B. B., \& Sandman, C. A. Influences of heart rate on the human visual evoked response. Journal of Comparative \& Physiological Psychology, 1979, 93, 717-729.

\section{REFERENCE NOTE}

1. Lacey, J. I. Personal communication, 1981.

(Manuscript received September 29, 1981; accepted for publication February 26, 1982) 
This document is a scanned copy of a printed document. No warranty is given about the accuracy of the copy. Users should refer to the original published version of the material. 